\title{
Dust in the Solar System
}

\author{
H. Fechtig \\ Max-Planck-Institut für Kernphysik, Heidelberg, FRG \\ Z. Naturforsch. 44a, 877-882 (1989); received May 16, 1989 \\ Dedicated to Heinrich Wänke on the occasion of his 60 th birthday
}

Properties of cometary dust particles are better known since the space missions to Comet Halley. Their properties (densities, atomic composition) are compared with relevant observations from lunar microcraters and in-situ experiments. At $1 \mathrm{AU}$ in the eliptic , 2/3 of the dust grains are normal density particles, presumably of asteroidal origin and irregularly shaped, while the remaining $1 / 3$ are low density particles, presumably of cometary origin, but due to solar irradiation in a processed state (corresponding to "Brownlee"-particles). Beyond the asteroidal belt only black cometary dust grains are observed which have recently been released from comet nuclei orbiting on highly eccentric trajectories.

Key words: Cometary dust, Core-mantle particles, Porous aggregates, Eccentric orbits, Asteroidal dust.

\section{Introduction}

The total amount of dust $(<100 \mu \mathrm{m}$ diameter $)$ in the solar system was estimated by Whipple [1] to be between 1.1 and $4.5 \times 10^{19} \mathrm{~g}$ (average $\sim 2.5 \times 10^{19}$ ) over a volume of $3.5 \mathrm{AU}$ radius (distance earth - sun 1 AU) about the sun within inclinations $<20^{\circ}$ relative to the ecliptic. A new estimate by Kneißel [2] with $2 \times 10^{19}$ g confirms Whipple's result.

Due to the Poynting-Robertson effect (Wyatt and Whipple [3]), the dust grains orbiting the sun continuously lose energy, thus spiralling slowly towards the sun. Dust grains also collide with each other. Particles which approach the sun close enough will be sublimated and their vapour finally becomes part of the solar wind.

The fact that the interplanetary dust cloud still exists after 4.6 billion years (Fig. 1 shows a photograph of the zodiacal light taken by Hutchison in 1967 at the Hawaii Observatory) necessarily means that there are dust sourcess. Whipple [1] has estimated that some 10 tons/s are necessary in order to maintain the zodiacal dust cloud in temporal equilibrium. Grün et al. [4] see the dust sources twofold: primary dust sources like comets or asteroids and a secondary dust source resulting from collisions between dust particles. Particularly the collision probability between dust grains increases with decreasing distance from

Reprint requests to Prof. Dr. H. Fechtig, Max-Planck-Institut für Kernphysik, Postfach 1039 80, D-6900 Heidelberg. the sun (Grün et al. [4]). Smaller dust particles are produced by collisions from larger ones. They either stay on bound orbits (so-called $\alpha$-meteoroids) and their diameters are generally $>1 \mu \mathrm{m}$ or are accelerated by solar pressure (generally diameters $<1 \mu \mathrm{m}$ ) thus leaving the solar system (so-called $\beta$-meteoroids).

The intention of this paper is to compare the new results of one of these sources, comets, with properties of interplanetary dust grains as seen in the study of lunar microcraters and in in-situ experiments. Suggestions are then being made for the evolution of dust grains in the solar system in keeping with the observations.

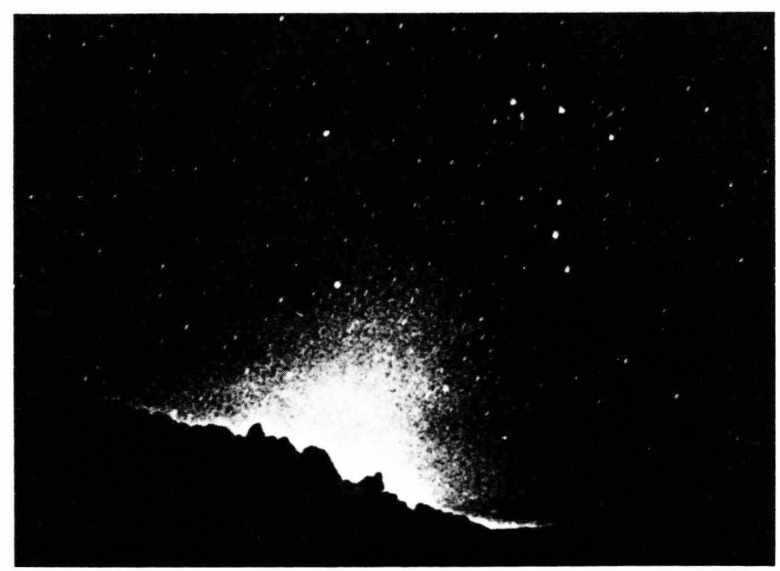

Fig. 1. Photograph of the zodiacal light (P. Hutchison, 1967, University of Hawaii).

0932-0784/89/1000-0877 \$01.30/0. - Please order a reprint rather than making your own copy. 


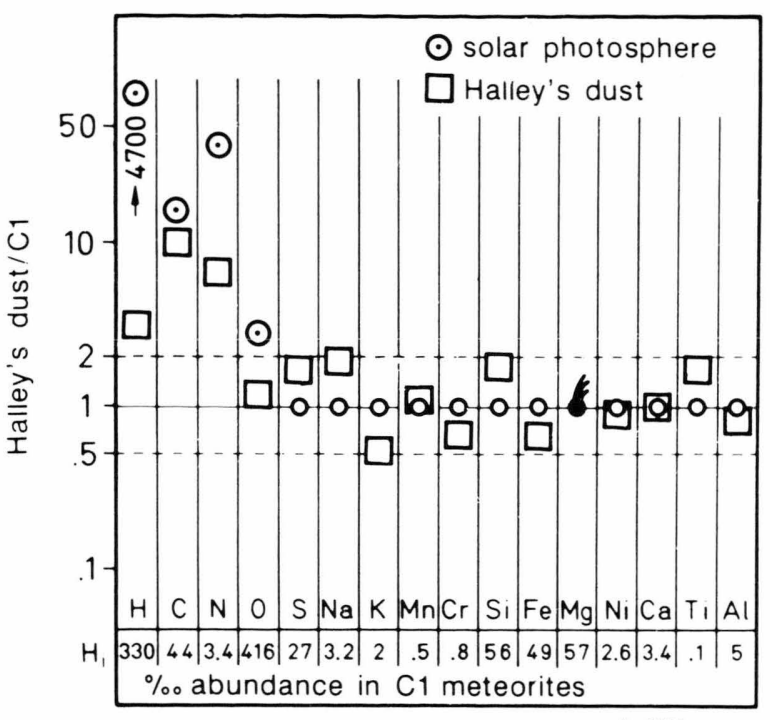

- volatility

Fig. 2. Atomic abundancies of Halley's dust in relation to $\mathrm{C} 1$ carbonaceous chondrites $\square$ and to the solar photosphere $\odot$ for the most abundant elements, normalized to $\mathrm{Mg}$.

\section{Properties of Halley Dust Particles}

In 1986 the cometary missions to Comet Halley obtained results on the elemental composition of Halley dust grains. The cometary source is now much better known than before. Here some basic results are summarized: Figure 2 shows the elemental abundances of the most frequent elements for Halley dust grains relative to $\mathrm{C} 1$ chondrites normalized to $\mathrm{Mg}$ [5]. For comparison the solar abundances for these elements are given, too. While the heavy elements agree within a factor of 2 between cometary dust grains and $\mathrm{C} 1$ chondrites, the light elements differ considerably. $\mathrm{H}, \mathrm{C}$ and $\mathrm{N}$ are enriched in cometary dust grains. Carbon is a factor of 12 more abundant in Halley dust than in $\mathrm{C} 1$ chondrites.

Because of the equal relative abundance of the heavy elements in cometary dust and $\mathrm{C} 1$ chondrites, one might assume that in addition to this silicate component, a second component is present consisting of light elements. This second component could potentially be cometary ice. Hanner [6] calculated how long cometary ice grains (or ice mixed with dust) ould exist after release from the comet nucleus as a solid. From these calculations one should expect that ice sublimates within a few minutes after release which corresponds to a travel distance of a few hundred kilome-

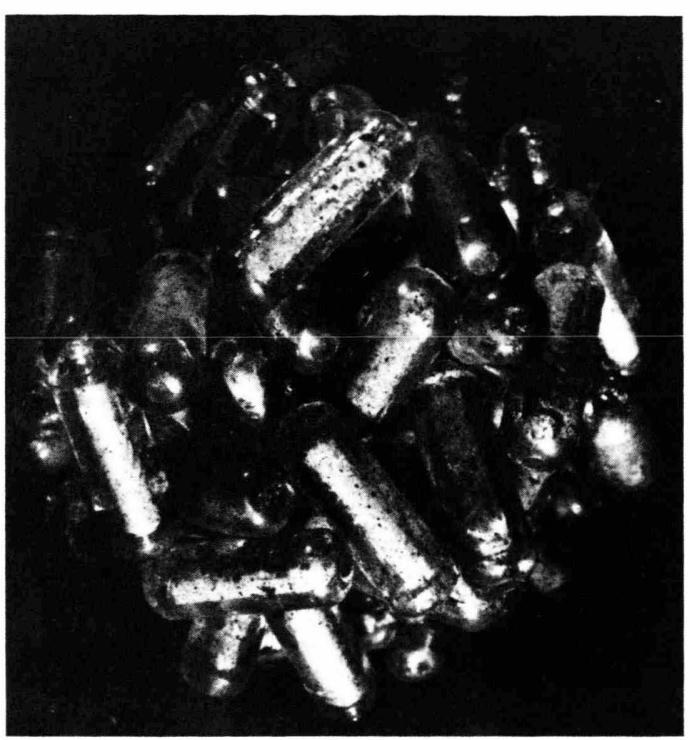

Fig. 3. Cometary model grain, suggested by Greenberg: conglomerate of elongated submicronsized building blocks with core/mantle structure.

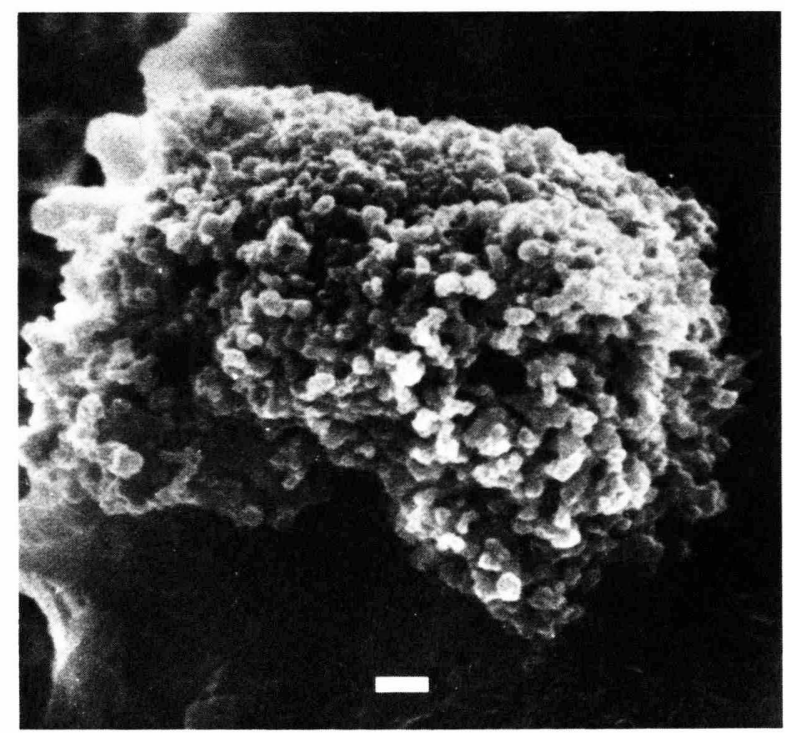

Fig. 4. Interplanetary dust particle, collected by Brownlee (socalled Brownlee-particle): a conglomerate of submicronsized building blocks (bar at bottom $=1 \mu \mathrm{m}$ ).

ters from the comet nucleus. If the second component is not cometary ice, what else could it be?

Greenberg [7] published his model dust particle based on simulation experiments. Figure 3 shows a photograph of the proposed cometary dust gain: it consists of loosely conglomerated building blocks. 
Each building block of submicronsize dimensions is elongated and shows a core-mantle structure. The cores are silicates and the mantles consist of organic molecules. If such a particle hits a metal target at a speed of $70 \mathrm{~km} / \mathrm{sec}$ (conditions during the Halley dust experiment), its mass spectrum will indeed show an elemental abundance digram similar to that given in Fig. 2 as a result of the Halley dust experiment.

Let us therefore assume that cometary dust particles are indeed structured as shown in Figure 3. The densities of Halley dust grains have been determined to be between 0.1 and $1 \mathrm{~g} / \mathrm{cm}^{3}$ [5], which is consistent with Greenberg's model particle. Porous particles were collected in the atmosphere by Bownlee [8]: Figure 4 is a photograph of one of these particles. This particle shows a similar structure as the Greenberg model grain, but the building blocks are generally not elongated. The elemental composition of Bownlee's most porous grains is identical to the silicate component of Halley dust particles. If these particles are of cometary origin, why do they not show the core/mantle configuration of the building blocks?

\section{Properties of Interplanetary Dust Particles at 1 AU}

Let us now compare the properties of cometary dust grains discussed above with properties of dust particles studied by various techniques in interplanetary space. Comparable data are available from studies of lunar microcraters and from dust experiments flown on the German-American space probe Helios and on the NASA deep space missions Pioneer 10 and 11.

Lunar microcraters were found in large numbers on many of the returned surface samples. They were produced by interplanetary dust impacts in size ranges between $0.1 \mu \mathrm{m}$ and $1 \mathrm{~mm}$, as shown in Fig. 5 on a glass-lined surface sample. The crater morphology has been characterized by the ratio of the crater diameter $D$ to the crater depth $T$, i.e. by $D / T$. Simulation experiments have shown that this ratio $D / T$ is independent of the projectile impact velocity (if $\geqq 5 \mathrm{~km} / \mathrm{sec}$ ) and of the projectile diameter between 0.1 and $100 \mu \mathrm{m}$ [9]. As shown in Fig. 6 (upper part) the dominating parameter for the diameter to depth ratio $D / T$ is the density of the projectile: for iron $D / T=1.3$, for silicastes (represented in the simulation experiments by glass projectiles) $D / T=1.85$ and, as shown by Mandeville [10], $D / T=2.5$ corresponds to a projectile density of ap-

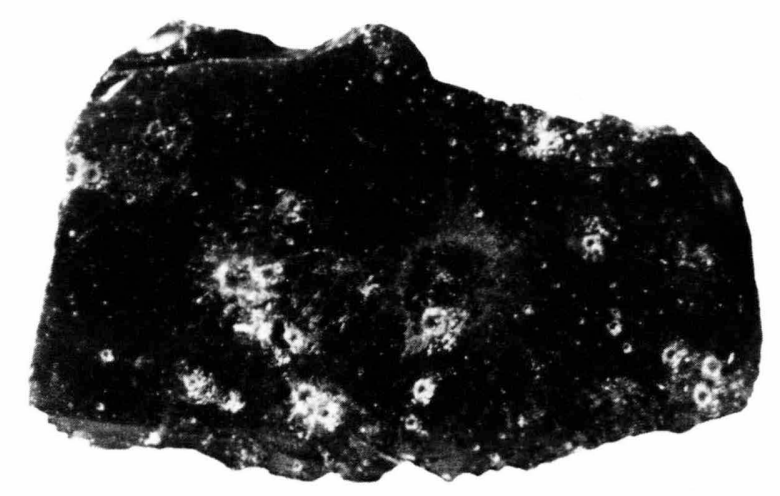

Fig. 5. Photograph of mm-sized impact craters on a glass lined lunar surface sample.
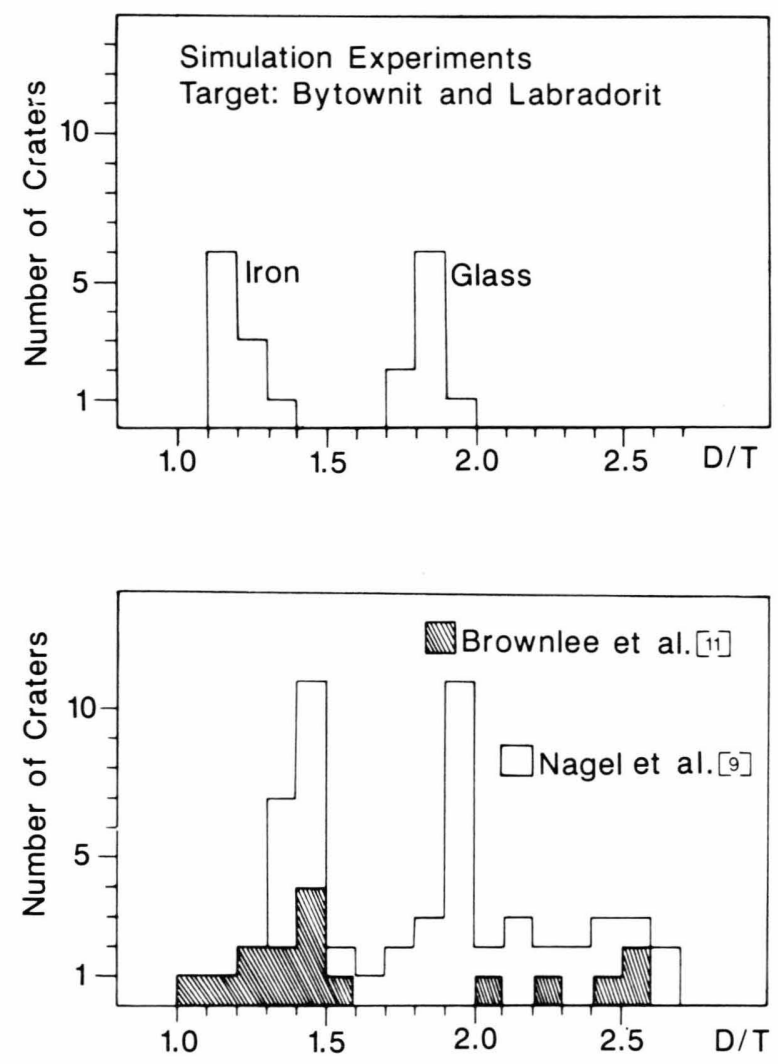

Fig. 6. Upper part: histogram of laboratory produced microcrater morphologies: diameter/depth ratio $D / T$ for iron and glass projectiles. Lower part: histogram of $D / T$ distribution of lunar microcraters. 
proximately $1 \mathrm{~g} / \mathrm{cm}^{3}$. From Fig. 6 (lower part) one concludes for the $D / T$ distribution of lunar microcraters $[9,11]$ that most projectiles are of densities between 3 and $8 \mathrm{~g} / \mathrm{cm}^{3}$ and only a smaller part $(\leqq 30 \%)$ of the projectiles shows a density as low as about $1 \mathrm{~g} / \mathrm{cm}^{3}$.

The sun probe Helios orbited the sun on a ellipse with apogee at $1 \mathrm{AU}$ and perigee at $0.3 \mathrm{AU}$ sun distance. The dust expriment consisted of 2 identical sensors which scanned in the ecliptic (so-called "ecliptic sensor") and in the southern polar direction (so-called "south sensor") [12]. For technical reasons the ecliptic sensor was covered by a $0.4 \mu \mathrm{m}$ thick penetration foil while the south sensor stayed open.

The results were quite surprising: contrary to all expectations the south sensor registered more dust particles than the ecliptic sensor [12], although the

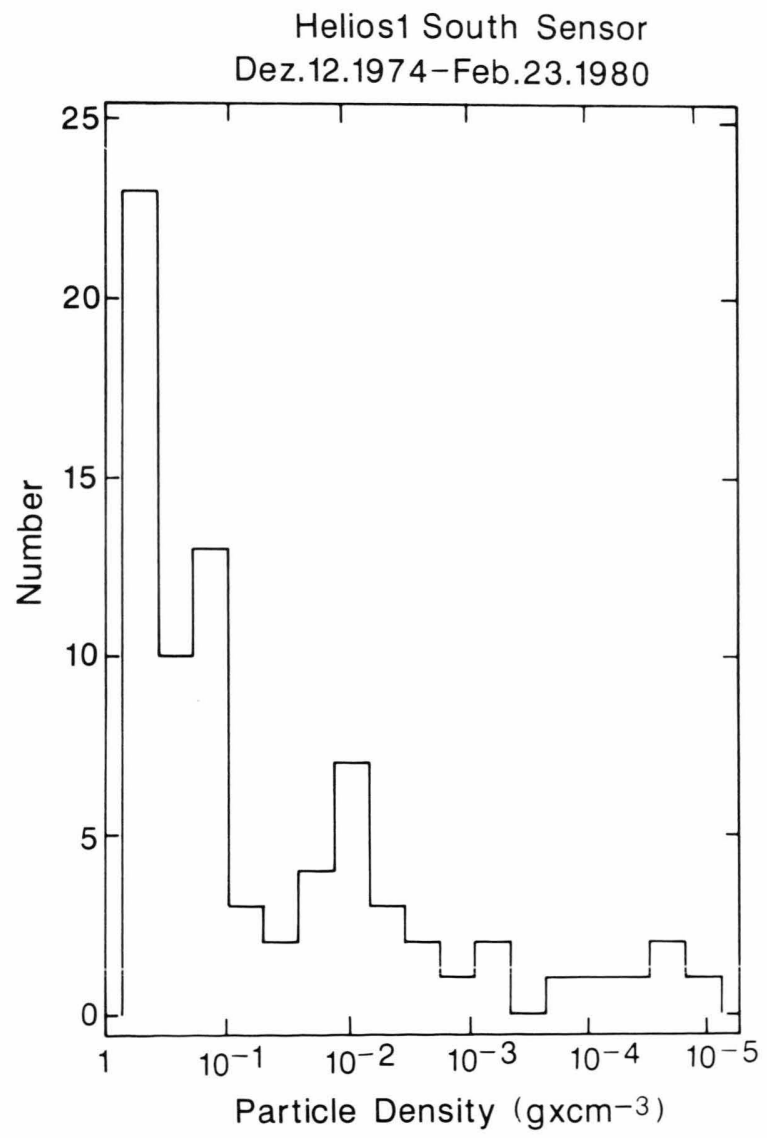

Fig. 7. Histogram of the "eccentric" particles registered by the Helios south sensor. The densities shown are extrapolated from penetration experiments with projectiles of densities $\geqq 1 \mathrm{~g} / \mathrm{cm}^{3}$. zodiacal light (Fig. 1) suggests that most particles orbit the sun at low inclinations to the ecliptic. The only explanation of this observation is that the cover foil of the ecliptic sensor prevented a number of particles to be registered. An experimental investigation of the cover foil [13] showed that low density dust grains are not able to penetrate the foil. The relevant result [12] was the discovery that 2 types of particles orbit the sun: those of "normal" densities (between 3 and $\left.8 \mathrm{~g} / \mathrm{cm}^{3}\right)$ on orbits with low eccentricities $(\varepsilon<0.6)$, and those of "low" densities $\left(\leqq 1 \mathrm{~g} / \mathrm{cm}^{3}\right)$ on orbits with high eccentricities $(\varepsilon>0.6)$. The latter component means porous (cometary) particles. Figure 7 shows a histogram of estimated particle densities for these "eccentric" particles. It is evident that the estimates are only qualitative and should not be taken quantitatively. A rough estimate shows that only $30 \%$ or less of all particles between 0.3 and $1 \mathrm{AU}$ sun distance are of low density and therefore of possible cometary origin.

The dust experiments on the space probes Pioneer 10 and 11 (a photometer and a penetration dust detector) have delivered results which seem to contradict each other. The optical photometer [14] registered the zodiacal light between 1 and $3.3 \mathrm{AU}$ sun distance (asteroidal belt) with decrease in intensity of the scattered sunlight when moving away from the sun. No increase of scattered sunlight was seen within the asteroidal belt. Beyond 3.3 AU sun distance, however, no scattered sunlight was measured, as originally expected. The penetration detector [15] recorded dust particles between 1 and $20 \mathrm{AU}$ sun distance [16]. Another important result (Humes [16]) is the fact that the particles beyond the asteroidal belt generally orbit the sun on highly eccentric, i.e. cometary-like trajectories. In order to explain the discrepancy outside the asteroidal belt between these 2 dust experiments, Cook [17] suggested that dust grains beyond the asteroidal belt may have extremely low albedos.

\section{Evolution of Cometary Dust Particles}

Following the thoughts of the chapter on properties of Halley dust particles and accepting Greenberg's model of core-mantle-particles as cometary grains, the question is, how cometary grains evolve after release from the comet nucleus.

The larger grains (generally $>1 \mu \mathrm{m}$ diameter) stay more or less on the orbit of the comet. During the 
course of time they form a meteor stream. In case of Comet Halley the associated meteor stream appears twice a year when the Earth travels through it in May (Eta-Aquarids) and in October (Orionids) [18]. For the grains below the threshold size (which depends on the orbital eccentricity of the parent comet) the radiation pressure of the sun dominates gravity: they form the dust tail of active comets and leave the solar system.

Dynamically the dust grains of meteor streams follow the Poynting-Robertson effect, i.e. dust grains spiral slowly (depending on their sizes) towards the sun. Therefore dust grains of Comet Halley experience a considerable temperature rise every orbit during perihelion passages. There are two main aspects to discuss:

a) The corpuscular irradiation (solar wind, cosmic rays) sputters first the molecular mantles of the cometary grains. Johnson and Lanzerotti [19] experimentally found that organic material under ion bombartment becomes dark because the carbon is sputtered away much slower than the other elements. Therefore cometary dust grains are black at the beginning, which means low albedos.

b) The electromagnetic solar irradiation determines the temperature of the cometary dust grains of the meteor shower. During the perihelion passages at 0.6 AU sun distance for Comet Halley the cometary grains reach temperatures of several hundred degrees. With decreasing sun distances due to the PoyntingRobertson-effect the temperatures become higher until the particles finally sublimate into the gas phase. It is clear that the organic mantle material will disappear first because it generally is not as resistent to high temperatures as silicates.

In an earlier paper [20] a possible scenario for the evolution of cometary dust grains was calculated. Black mantle-core grains ("young" cometary grains) as shown in Fig. 8 (left) lose organic molecules on the uppermost layer of a conglomerate cometary particle. At an intermediate state (Fig. 8, middle) the uppermost layers have lost their mantles completely: the silicate cores with higher albedos stick on the black building blocks due to the packing effect as discussed in [20]. And finally only the silicate cores remain as a conglomerate dust grain (Fig. 8, right). In reality, the silicate cores are still coated with a thin layer of mainly carbon, as shown by Wopenka [21]. Figure 9 shows how the density changes as function of time for a $100 \mu \mathrm{m}$ sized grain for loss rates of $5 \cdot 10^{-13}, 1 \cdot 10^{-13}$ and $0.5 \cdot 10^{-13} \mathrm{~g} \mathrm{~cm}^{-2} \mathrm{sec}^{-1}[20]$. Within $10^{5}$ years
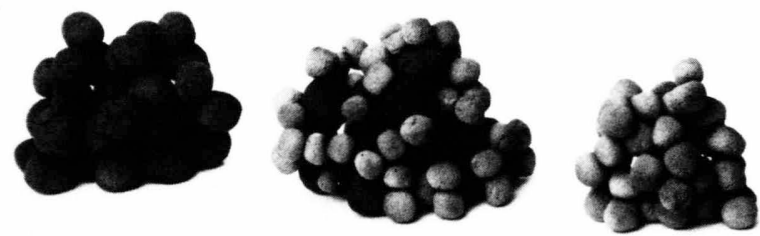

Fig. 8. Cometary model grains. Left: "young" grain: porous conglomerate of black mantled building blocks. Middle: "intermediate" grain: silicate cores (bright color) stick to black mantled grains. Right: "old" grains: porous conglomerate of silicate cores (correspondent to Brownlee-particles).

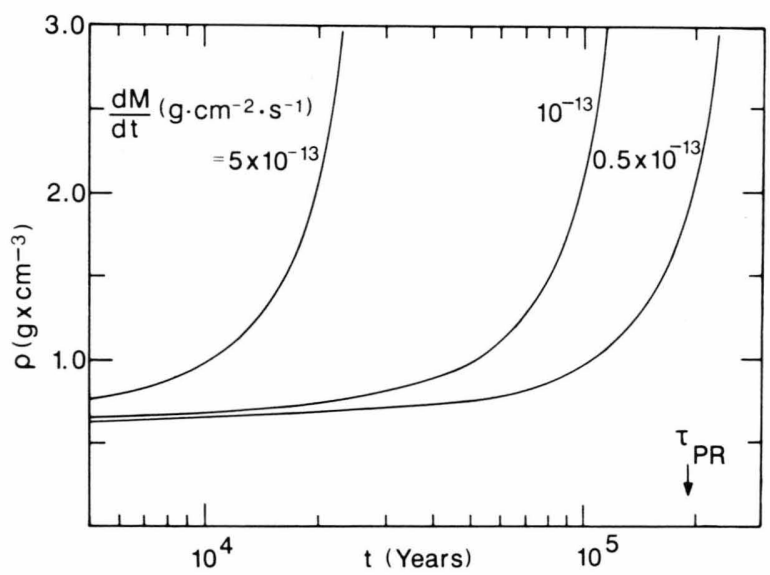

Fig. 9. Density varition vs. time for a $100 \mu \mathrm{m}$ sized Halley dust grain after release from comet nucleus for 3 different mass loss rates. $\tau_{\mathrm{PR}}$ indicates the time when the particle orbit is of low eccentricity due to the Poynting-Robertson effect.

the density might have been increased due to the packing effect from 0.6 to $2 \mathrm{~g} \mathrm{~cm}^{-3}$. These $10^{5}$ years, however, are comparable with the time needed by the orbits to become quasi circular (far within the asteroidal belt) due to the Poynting-Robertson effect (as indicated in Fig. 9 by $\tau_{\mathrm{PR}}$ ).

As a consequence of these evolution processes "young" cometary grains with a core-mentle structure may change into "old" cometary grains which Brownlee collected and investigated [8]. It is also possible that by this evolution amorphous grain material may become crystalline by phase changes as observed in collected dust grains. 


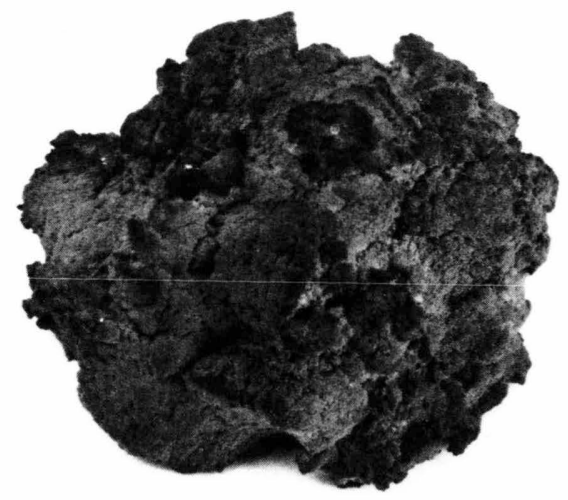

Fig. 10. Asteroidal model grain: irregularly shaped, 10 to $20 \%$ albedo.

\section{Summary}

Comparing the observations discussed above with the properties of "young" and "old" cometary dust grains the following expectations can be stated:

[1] F. L. Whipple, On Maintaining the Meteoritic Complex, in: The Zodiacal Light and the Interplanetary Medium (J. L. Weinberg, ed.), NASA SP-150, 409-426 (1967).

[2] B. Kneißel, personal communication (1988).

[3] S. P. Wyatt and F. L. Whipple, Atrophys. J. 111, 134 (1950).

[4] E. Grün, H. A. Zook, H. Fechtig, and R. H. Giese, Icarus 62, 244 (1985).

[5] E. K. Jessberger, J. Kissel, H. Fechtig, and F. R. Krueger, On the average chemical composition of cometary dust, ESA SP-249, 27 - 30 (1986).

[6] M. S. Hanner, Icarus 47, 342 (1981).

[7] J. M. Greenberg, Laboratory dust experiment - tracing the composition of cometary dust, in: Cometary Exploration (T. L. Gombosi, ed.), Hungarian Academy of Sciences, Budapest, Vol. II, p. 23 (1983).

[8] D. E. Brownlee, Microparticle studies vby sampling techniques, in: Cosmic Dust (J. A. M. McDonnell, ed.), Chichester, Wiley \& Sons, New York 1978, p. 295.

[9] K. Nagel and H. Fechtig, Planet. Space Sci. 28, 567 (1980).

[10] J.-C. Mandeville, Impact microcraters on 12054 rock (Ed. R. B. Merril, Houston, Texas), Proc. Lunar Sci. Conf. 8th, Pergamon Press, New York 1977, p. 883 (Ed. R. B. Merrill, Houston, Texas).
Beyond the asteroidal belt $(>3.3 \mathrm{AU})$ all dust grains are black and orbit the sun on highly eccentric trajectories. This corresponds ideally to the properties of "young" cometary dust grains as modelled by Greenberg [7] (Fig. 8, left).

Between the sun and the asteroidal belt $(<3.3 \mathrm{AU})$ there are 3 different types of particles present. Firstly the "young" cometary grains, which stay only a short fraction of time in this space (due to their highly eccentic orbit); secondly the "old" cometary grains (Fig. 4 and Fig. 8, right) and thirdly particles with densities between 3 and $8 \mathrm{~g} \mathrm{~cm}^{-3}$, most likely of asteroidal origin.

All cometary grains between 1 and $3.3 \mathrm{AU}$ (between Earth and the asteroidal belt) make up only about $1 / 3$ of all grains, while the asteroidal grains make up $2 / 3$. These abundant asteroidal grains are produced by collisions between asteroidal bodies within the asteroidal belt. Figure 10 shows a photograph of a model asteroidal particle: it is presumably of irregular shape and should have a "normal" albedo between 10 to $20 \%$. The existence of asteroidal dust has been observed by the IRAS-satellite [22]. More investigations of the dynamics of asteroidal dust is needed.

[11] D. E. Brownlee, F. Hörz, J. F. Vedder, D. E. Gault, and J. B. Hartung, Some physical parameters of micrometeoroids (Ed. W. A. Gose, Houston, Texas), Proc. Lunar Sci. Conf. 4th, Pergamon Press, New York 1973, p. 3197 (Ed. W. A. Gose, Houston, Texas).

[12] E. Grün, N. Pailer, H. Fechtig, and J. Kissel, Planet. Space Sci. 28, 333 (1980).

[13] N. Pailer and E. Grün, Planet. Space Sci. 28, 321 (1980).

[14] M. S. Hanner, J. C. Sparrow, J. C. Weinberg, and D. E. Besson, Pioneer observations of zodiacal light brightness near the ecliptic: changes with heliocentric distances, in: Lecture Notes in Physics (H. Elsässer and H. Fechtig, eds.), Springer-Verlag, Berlin 1976, Vol. 48, p. 29.

[15] D. H. Humes, J. D. Alvarez, R. L. O'Neil, and W. H. Kinard, J. Geophys. Res. 79, 3677 (1974).

[16] D. H. Humes, J. Geophys. Res. 85, 5841 (1980).

[17] A. F. Cook, Icarus 33, 349 (1978).

[18] D. W. Hughes, Astron. Astrophys. 187, 879 (1987).

[19] R. E. Johnson and L. J. Lanzerotti, Icarus 66, 619 (1986).

[20] T. Mukai and H. Fechtig, Planet. Space Sci. 31, 655 (1983).

[21] B. Wopenka, EPSL 88, 221 (1988).

[22] S. F. Dermott, P. D. Nicholson, J. A. Burns, and j. R. Houck, Nature London 312, 505 (1984). 